
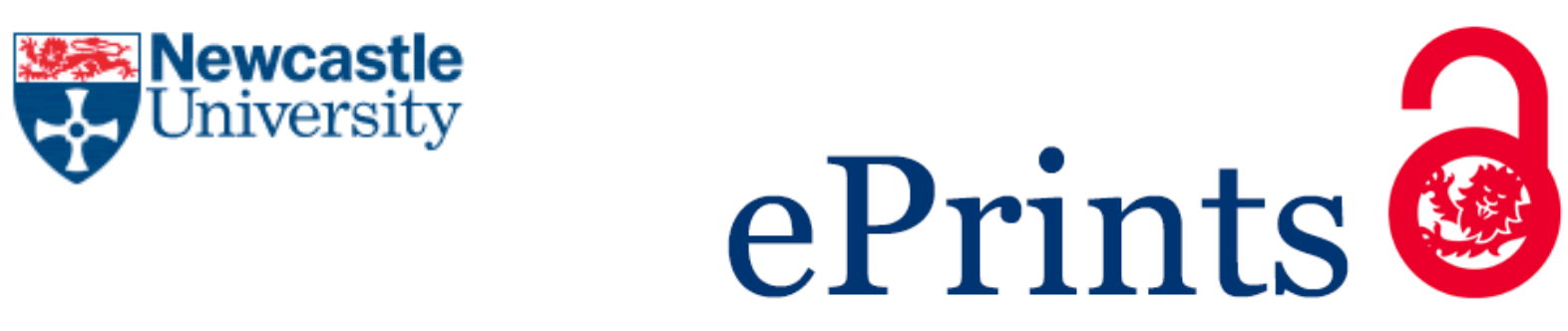

Miles P, Cassidy P, Donlon L, Yarkoni O, Frankel D. In Vitro assembly of a viral envelope. Soft Matter 2015, 11(39), 7722-7727.

\title{
Copyright:
}

C Royal Society of Chemistry 2015

DOI link to article:

http://dx.doi.org/10.1039/C5SM01695A

Date deposited:

$01 / 10 / 2015$

Embargo release date:

19 August 2016

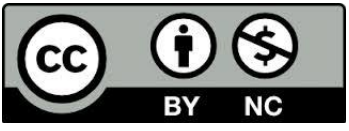

This work is licensed under a Creative Commons Attribution-NonCommercial 3.0 Unported License 


\section{In vitro assembly of a viral envelope}

\section{Penny Miles ${ }^{\mathrm{a}}$, Peter Cassidy ${ }^{\mathrm{a}}$, Lynn Donlon $^{a}$, Orr Yarkoni $^{\mathrm{a}}$ and Daniel Frankel ${ }^{*, a}$}

Viruses such as Influenza and Ebola are enveloped in lipid bilayers annexed from host cells and 5 containing glycoproteins essential for the infection process. At the molecular level little is known about the assembly process in terms of physical interactions between the lipids and glycoproteins. In this paper we assemble HIV glycoproteins in lipid vesicles in order to examine envelope assembly, a process that is usually only executed under control of a host cell. Using atomic force microscopy it was possible to observe fusion of individual envelope like particles, and contrast this with the behaviour of lipid vesicles 10 without envelope glycoproteins. It was found that the inclusion of glycoproteins caused the vesicles to distort and that the subsequent fusion "footprint" with a lipid bilayer was related to the envelopes' unique morphology. This non- spherical morphology suggests that the presence of a viral capsid may be essential for the stability of an enveloped virus. Interactions between trans-membrane gp41 and gp120, the spikes protruding from a virion, were examined using supported lipid bilayers. Interactions between the gp120

15 and membrane-located gp41 resulted in the assembly of unusual molecular wires, one molecule in height and with a zigzag arrangement of gp120 molecules. In this work we have shown that purely physical/chemical interactions have dramatic effects on glycoprotein/lipid assembly and should be considered in the development of virus based technologies such as virosomes.

\section{Introduction}

20 Viruses including Ebola and HIV are enveloped in a lipid bilayer, forming a soft interface between a virus particle and its environment. Typically the viral envelope is composed of lipids from the cell membrane of its host, which it annexes when the virion buds from the cell membrane. ${ }^{1}$ In addition to the lipid 25 components, the virus genome synthesises glycoproteins, which are inserted into the bilayer. These glycoproteins mediate the recognition and fusion between the virus and the host, allowing infection of a healthy cell with the viral genome. In the case of HIV, glycoproteins are derived from the polypeptide gp160, 30 which is coded for by the env gene. This precursor then trimerizes in the endoplasmic reticulum, before being cleaved by furin proteases to form the gp120-gp41 complex. This complex is then transported to the cell surface via the Golgi, where the virion

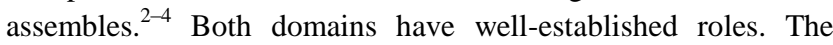

35 gp41 domain mediates the fusion between the virion and the target cell, whilst the gp120 domain protrudes from the lipid envelope and plays a crucial role in attachment to the CD4 receptor on target cells. ${ }^{5,6}$ As such, the gp120 domain has long been seen as one of the most promising targets for a HIV vaccine, 40 and indeed neutralizing antibodies have been shown to act by obstructing the interaction between gp120 and CD4 receptors. ${ }^{7}$

These efforts however have been held back by obscurities in the conformational changes that take place during the receptor binding and fusion process. As a result the structure of the gp12045 gp41 heterodimer has only recently been reported. ${ }^{8}$ Extensive amounts of rapidly evolving N-linked glycosylation to the gp120gp41 complex also obscure the sites of interest. As the bond between gp41 and gp120 is non-covalent, it is relatively weak, thus when it is isolated the complex is unstable. ${ }^{9}$

50 As well as the relationship between lipids and glycoproteins, the composition of lipids in the envelope play an important part in envelope assembly and function. It has been hypothesised that organised gel-phase domains of phase-separated membranes play significant roles in cellular processes. If such membrane 55 inhomogeneity is present in the viral envelope it could also affect the assembly process. The potential involvement of gel-phase domains in viral processes is consistent with the observation that virus budding from a host cell only occurs from specific compositions of the host cell membrane. ${ }^{10}$ In addition host cell 60 lipid rafts have been shown to have a role in the budding process of the immature HIV virion, which have an analogous structural organization to the gel-phase domains. ${ }^{11}$

Atomic force microscopy (AFM) and single-molecule force spectroscopy have become useful tools for investigating the 65 interactions of biomolecules with supported lipid bilayers (SLBs), simplified models of cell membranes. ${ }^{12}$ These models possess the advantage of allowing physical phenomena such as selfassembly and interactions to be dissociated from the biological. An added advantage of using AFM to examine model bilayer 70 systems is that assembly processes can be followed with nanometre resolution under aqueous conditions. One such membrane system that incorporates complexity via phase separation of lipids is that of DOPC/DPPC, whereby the DPPC forms gel-phase islands in a liquid disordered DOPC phase. In 75 this context HIV glycoprotein-lipid interactions have been probed with the ability to observe nanometre scale resolution in real time under physiologically relevant conditions. ${ }^{13-15}$

In this work glycoprotein lipid interactions have been examined in order to better understand the assembly of the HIV 80 envelope. Virion assembly is usually completed under direction of the host cell machinery, however an in vitro approach has the advantage of being able to elucidate the purely physical/chemical interactions of the biomolecular components. AFM was used to follow the fusion of gp160 reconstituted lipid vesicles, as mimics 85 of the viral envelope, as they formed a supported lipid bilayer. Vesicles were extruded through $100 \mathrm{~nm}$ pores to obtain uniform vesicles, $100 \mathrm{~nm}$ in diameter, representative of the approximate $120 \mathrm{~nm}$ diameter of the HIV virion. ${ }^{16}$

Significant differences in both morphology and fusion 
behaviour were noted as a consequence of the presence of the gp160. In order to investigate the nature of the gp120-gp41 complex, responsible for the "spikes" protruding from the HIV virion, gp41 was incorporated into phase-separated SLBs. Gp120 5 was then adsorbed from solution onto this gp41-containing supported lipid bilayer. Molecular wires were formed when gp120 was adsorbed from solution onto the gp41 containing bilayer, further demonstrating the intricacies of lipid/glycoprotein interactions in the assembly process.

10 This observation has relevance for supramolecular chemistry, as exploiting this reversible host-guest interaction could be used to design nano-assemblies. A similar process has driven a strand of research focusing on cyclodextrin-based assemblies, a potential strategy applicable to the gp41-gp120 complex. ${ }^{17,18}$ 15 Virosomes are nanoparticles that contain inactive versions or fractions of a virus. They are used to elicit an immune response, generating antibodies against the viral antigen. Incorporating $t$ host-guest interaction strategies into virosome development could lead to improved presentation of glycoproteins, giving a better 20 chance of vaccine like effects.. This could be especially beneficial in the case of HIV as no effective vaccine exists.

\section{Materials and Methods}

\section{HIV gp160 and gp41 reconstitution into vesicles}

Incorporation of the HIV glycoproteins into vesicles and 25 subsequent bilayer formation was achieved via reconstitution of the glycoproteins into lipid vesicles followed by extrusion resulting in unilamellar vesicles. Either HIV gp160 (ab73770, Abcam, UK) or gp41 (ab49070, Abcam, UK), depending on the experiment, were diluted in ultrapure water to a working 30 concentration of $20 \mu \mathrm{g} \mathrm{mL}^{-1}$. A 3:1 molar ratio of 1,2-dioleoyl-snglycero-3-phosphocholine (DOPC) and 1,2-dipalmitoyl-snglycero-3-phosphocholine (DPPC) (Avanti Polar Lipids) were dissolved in chloroform followed by drying under nitrogen. The dried lipid film was then re-suspended in ultrapure water to give a 35 final concentration of $1 \mathrm{mg} \mathrm{mL}^{-1}$. DOPC/DPPC vesicles were then subjected to 10 freeze-thaw cycles. The relevant glycoprotein (gp160 or gp41) was then added to the vesicle solution giving a protein concentration of $1 \mu \mathrm{g} \mathrm{mL} \mathrm{m}^{-1}$. Following this the vesicles were extruded at $45^{\circ} \mathrm{C}$ ten times through a polycarbonate 40 membrane (pore size 100nm) by means of a mini extruder (Avanti Polar Lipids).

\section{Supported lipid bilayer formation}

Supported lipid bilayers were prepared via vesicle fusion. $400 \mu \mathrm{L}$ of the liposome solution was pipetted onto freshly cleaved 45 mica and allowed to anneal for 20 minutes at $45{ }^{\circ} \mathrm{C}$. The surface was then gently washed 3 times with PBS to remove nonabsorbed vesicles prior to imaging. ${ }^{19-21}$

\section{Gp120 Adsorption}

For experiments where gp120 was adsorbed from solution onto 50 a gp41-reconstituted bilayer, the gp120 (ab73769, Abcam, UK) solution was added to the preformed SLB and allowed to equilibrate for 60 minutes.

Topographic AFM imaging
For images of vesicles interacting with the surface and forming 55 a bilayer the standard annealing step was significantly shortened to 5 minutes. AFM measurements were performed in a liquid cell using an Agilent 5500 AFM microscope. Images were obtained in both contact and tapping mode. Contact mode images were obtained using nitrogen doped silicon tips with a nominal force 60 constant of 0.02-0.77. Tapping mode images were obtained using silicon tips (Nanosensors series PPP-NCH) with a resonance frequency $\sim 330 \mathrm{kHz}$ and a force constant of $0.42 \mathrm{~N} \mathrm{~m}^{-1}$. Forces were minimized during scans remaining below $1 \mathrm{nN}$, with typical scan rates in the range of $0.5-1 \mathrm{kHz}$ at 512 -pixel resolution. All 65 measurements were performed in phosphate buffered saline (PBS) solution at ambient temperature $\left(\begin{array}{rll}{ }^{\circ} \mathrm{C}\end{array}\right)$. Image processing was performed using version 5.1.6 of the Scanning Probe Image Processor (SPIP) software (Image Metrology, Lyngby, Denmark).

\section{${ }_{70}$ Force spectroscopy}

Force spectroscopy experiments were conducted using backside aluminium coated silicon cantilevers (PPP-CONTR, Nanosensors, Switzerland) with a nominal spring constant of 0.02 - $0.77 \mathrm{~N} \mathrm{~m}^{-1}$ and typical tip radius of less than $7 \mathrm{~nm}$. Spring 75 constants were calibrated using the equipartition theorem (Thermal K) as described by Hutter and Bechhoefer. ${ }^{22}$ At least 1200 force-distance curves were obtained per sample with an applied load in the range of 9-10 nN, curve length of $1 \mu \mathrm{m}$ and constant tip velocity of $1 \mu \mathrm{m} \mathrm{s}^{-1}$. Force spectra were analysed 80 (using SPIP) to obtain unfolding forces. Only curves displaying well-defined sawtooth patterns that could be fitted to the wormlike chain model were attributed to single molecule pulling events.

\section{Results and Discussion}

85 As a control the fusion behaviour of vesicles consisting of lipids only, i.e. without reconstituted gp160, was studied. Vesicles were composed of a binary mixture of DOPC and DPPC in a molar ratio of $3: 1$. Observation of fusing vesicles revealed the characteristic annular shape previously reported for vesicles 90 collapsing on a surface (Fig. 1a). It is possible to resolve individual vesicles losing their individuality as they are incorporated into the lipid bilayer. The individual vesicles have a spherical morphology and a height of between 8 and $15 \mathrm{~nm}$. These structures have been investigated thoroughly by Jass et al. ${ }^{23,24}$ ${ }_{95}$ Eventually the fused vesicles form a complete bilayer (Fig. 1b), with island-like features corresponding to the gel phase domains. These are characteristic of phase separation and have a height between $1-2 \mathrm{~nm}$ relative to the surrounding liquid disordered phase. 

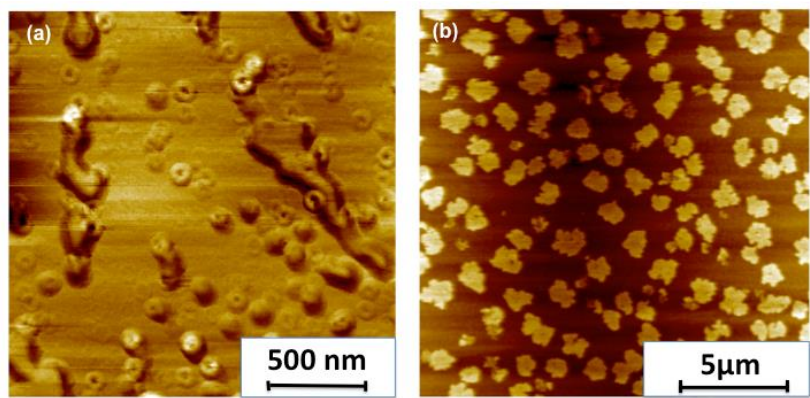

Fig. 1. Atomic force microscopy images showing fusion of lipid vesicles to form a phase separated supported lipid bilayer. Bilayer imaged with a short annealing phase (5 minutes), allowing individual vesicles to be 5 observed (a). Bilayer imaged with 20-minute annealing time, showing formation of a phase separated bilayer with typical gel phase islands (DPPC) surrounded by liquid disordered DOPC (b).

Vesicles reconstituted with gp160 were then deposited on a mica surface, and imaged quickly after formation in order to 10 observe the intact envelope-like particles fusing with each other and the surface. The particles can be described as envelope-like as they are composed of a lipid bilayer with the HIV envelope glycoprotein reconstituted into this membrane, a simplified model of the HIV lipid envelope. For comparison lipid vesicles forming 15 a bilayer are presented in Fig. 2a. Even at this resolution there is a striking difference in particle morphology between the HIVgp160 vesicles (Fig. 2b) and the control lipid only vesicles.
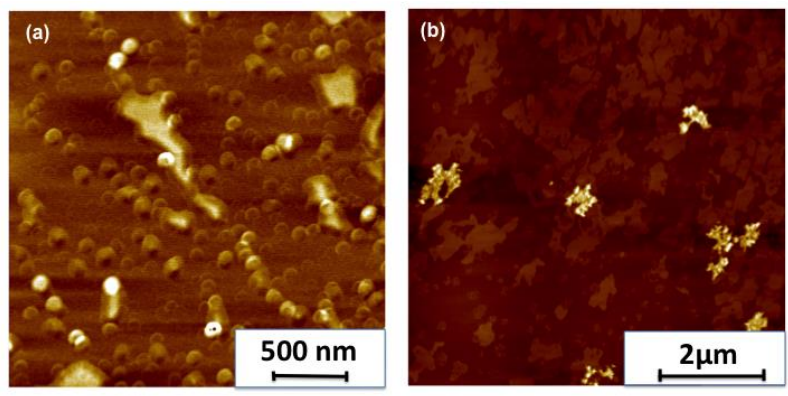

Fig. 2. Comparison between plain lipid vesicles and envelope-like 20 particles. DOPC/DPPC vesicles adsorbing onto the bilayer. These are round, uniform in size and of the correct dimensions to be single vesicles (a). Envelope-like particles, DOPC/DPPC vesicles reconsituted with gp160, adsorbing onto the bilayer. These are too large to be of single vesicle size and have a distinct morphology.

${ }_{25}$ It is clear that the gp160-laden vesicles form a larger, more disorganized structure in comparison to the small spherical vesicles containing only DOPC/DPPC. At higher resolution the distinct morphology (and size) of the envelope-like particles is obvious (Fig. 3b), in comparison to the individual lipid vesicles 30 (Fig. 3a).

There are two characteristic features in an individual HIV envelope-like particle (Fig. 3b), bright spots, with the brightness indicating height, and a less intense matrix. The bright spots are uniform in both height and size. The spots themselves are too

35 small to be individual vesicles (vesicles having been extruded through $100 \mathrm{~nm}$ pores), however they do appear to form a part of aggregated structures. Many of the aggregates show a 3-fold or 6fold assembly, which was previously reported for naked gp160 on mica. $^{25}$
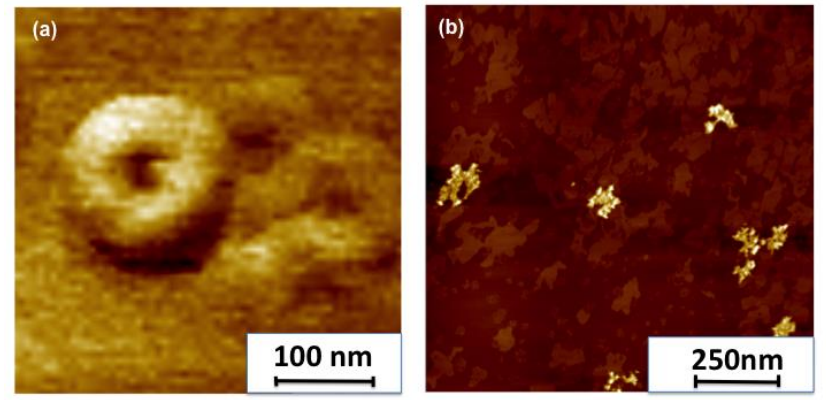

Fig. 3. Comparison of the difference in features. Lipid only vesicles. These have a symmetrical doughtnut like structure on contact with the underlying bilayer, indicative of a sphere collapsing onto a surface (a) gp160 laden vesicles with no symmetry. The bright features are thought to 45 be protruding gp160 molecules/clusters, within a lipid matrix (b)

These observations and the obvious differences between glycoprotein containing and control vesicles suggest that the incorporated glycoproteins distort the lipid vesicle shell, the protruding glycoproteins clearly visible. Given the size of the 50 individual envelope like particles larger than the $100 \mathrm{~nm}$ extrusion pore, it is possible that the glycoproteins have caused particle aggregation. This is not implausible as virus aggregation has been documented for HIV virus particles in vivo and has shown a relationship with infectivity. ${ }^{26}$ Confirmation of the protein nature 55 of the bright features was obtained using force microscopy.

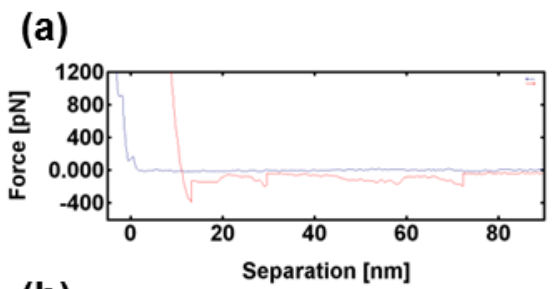

(b)

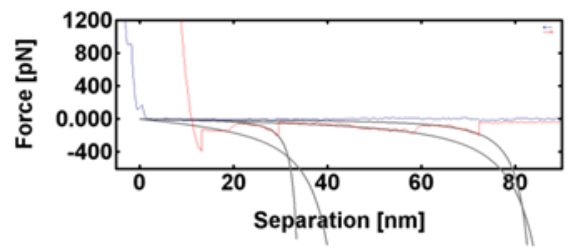

Fig. 4, Force-separation profile of the bright features within an envelopelike particle. A typical retraction curve shows multiple unfolding events indicative of the unfolding of a single protein molecule, in this case 60 attributed to the unfolding of gp160 (a). A worm like chain fit to sawtooth features in the retraction curve (b)

A typical force-separation profile of the bright features (Fig. 4a) shows multiple unfolding events characteristic of the unfolding of protein domains. Each of these unfolding events could be fitted to ${ }_{65}$ the worm like chain model (Fig. 4b), allowing the unfolding forces to be extracted. The form of the retraction curve and magnitude of the unfolding forces are characteristic of HIV- 
gp160 measured previously. ${ }^{25}$ This non-spherical morphology due to the inclusion of glycoproteins suggests that a protein capsid may be essential for the HIV virion to retain a spherical structure. Such an inference would be critical in the design of 5 virosomes towards a HIV vaccine. Indeed virosomes incorporating gp41 have shown promise in recent years as an immunization strategy, thus their in vitro self-assembly properties when reconstituted into liposomes are of great interest. ${ }^{27}$ In addition, a major issue in the targeting of the gp41-gp120 10 complex for vaccine design is the non-native forms of the Env gene that obfuscate the neutralization of the fusogenic form of Env. Unprocessed gp160, gp41 stumps and shed gp120 molecules have all been found to complicate the immune response. Hence an understanding of how the behaviour of these membrane 15 proteins translates to a virosome system is an important factor in rationalizing their design. ${ }^{28-30}$

Following the initial fusion of the gp160-laden vesicles with the supported lipid bilayer, the footprint of the fusion product could be seen to rearrange over time. From something resembling the 20 initial envelope-like particle (Fig. 5a) to more ring-like structures (Fig. 5b), and finally domains that resemble the gel-phase in a phase separated bilayer. This is particularly interesting as it reveals how envelope-like particle morphology is preserved in the fusion process and rearranges over time. This can be a useful 25 model for the fusion process of virion particles.
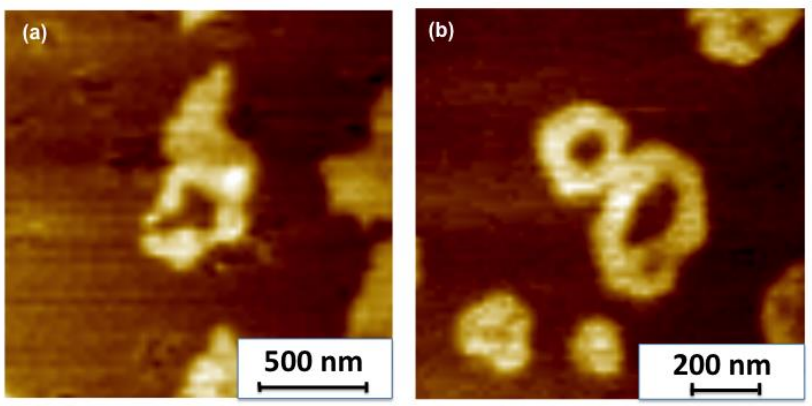

Fig. 5. Following fusion with the bilayer, the gp160-loaded vesicles leave a characteristic footprint that differs considerably from the naked vesicle doughnut shape. Initial footprint (a). Rearrangement after 20 minutes (b).

30 Lipid bilayers incorporating only the transmembrane gp41 section of the gp160 glycoprotein were then examined as a prelude to studying gp120-gp41 interactions. Topographic images present a similar landscape to the DOPC/DPPC only system with gel phase domains clearly defined. However, very small 35 individual features can be observed in between the gel phase domains in the liquid disordered regions of the membrane (Fig 6a). Given that such features are absent in the control DOPC/DPPC systems we assign these to be individual molecules or clusters of the transmembrane receptors gp41. In addition to 40 these randomly distributed gp41 molecules, the height of the phase separated gel domains increase in some cases giving a mean feature height of $3.15 \mathrm{~nm}$, significantly higher than the approximate $1 \mathrm{~nm}$ of the DPPC gel domain relative to the surrounding DPPC. This is consistent with previous studies 45 having reported the size of the gp41 molecule as $8.8 \mathrm{~nm}$, causing the overall height of the bilayer to be $5-6 \mathrm{~nm}^{31,32}$ It is also possible to observe "streaky" gel phase domains (Fig. 6b) where only parts of the gel-phase domain contain gp41. Therefore it can be concluded that gp41 will partition into both the liquid ${ }_{50}$ disordered and the gel phase domains.
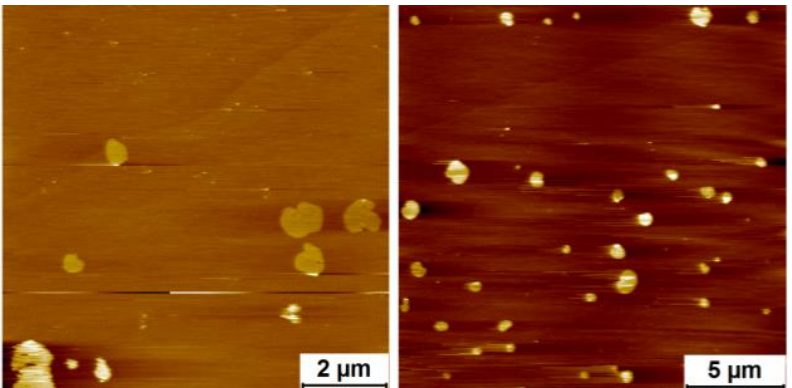

Fig. 6. Gp41 incorporated into a DOPC/DPPC bilayer. Gp41 can be observed randomly distributed in the liquid disordered phase (a). The transmembrane glycoprotein is also apparent in the gel phase domains, as 55 characterised by non-uniform, height features on the gel-phase domains.

Gp120 was then adsorbed onto the bilayer surface in order to probe interactions with gp41 incorporated into the lipid bilayer. Immediately apparent from the AFM topography images were the presence of wire-like features, microns in length and nanometres 60 in width (Fig. 7a, 7b). These wire features only form when gp41 is incorporated in the bilayer, with gp120 exhibiting a random orientation in the absence of the trans-membrane glycoprotein. These wires only form in the presence of both gp120 and gp41, without the gp41 the glycoproteins adopt a random distribution. ${ }^{33}$ ${ }_{65}$ On closer inspection, individual molecular features can be resolved (Fig. 8a) confirming the molecular nature of the wires. The deflection signal from the contact mode imaging, allows the extent of wire formation to be appreciated (Fig. 8b).
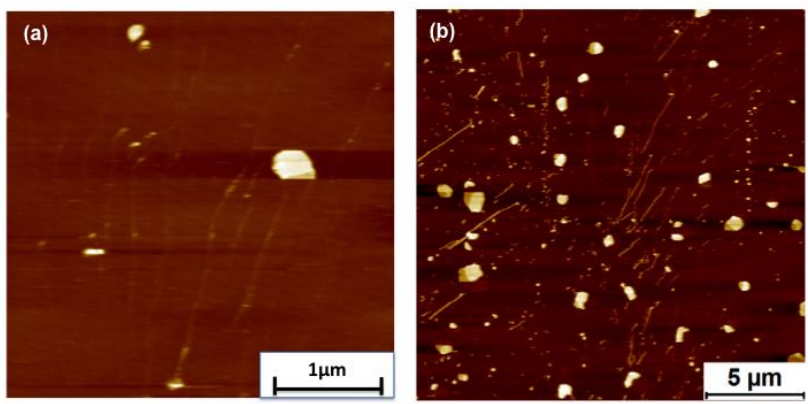

70 Fig. 7. Gp120 adsorbed onto supported lipid bilayers containing gp41. Molecular wires microns in length have assembled and decorate the bilayer surface (a). Features are reproduced across the lipid bilayer with varying orientations (b).

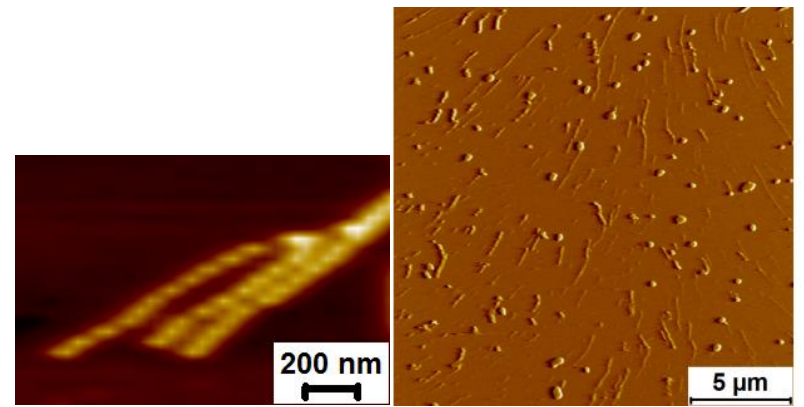


Fig. 8. Self-assembled molecular wires. Internal structure of wires cropped but not zoomed in from fig $7 \mathrm{~b}$ (a). Deflection image of selfassembled wires presenting extent of formation (b).

The dimensions of the wires are consistent with single molecule 5 heights, and multimer widths, fig. $9 \mathrm{a}$ and fig. $9 \mathrm{~b}$. These wires also assembled when a gold tip was functionalised with gp120 and scanned over the gp41 incorporating bilayer. Thus the most likely explanation for their assembly is that the non-covalent interactions responsible for holding the gp41-gp120 complex 10 together compete with gp120 aggregation interactions causing directional assembly to occur. In addition wires appear to exhibit a chirality, with one of two orientation, one a reflection of the other. These preferred orientations are particularly clear in figure $8 \mathrm{~b}$. Whether or not such structures can assemble in a biological 15 context is debatable, however the wire formation does demonstrate the directional and competitive aspect of intermolecular forces and how these could play a role in virion assembly.
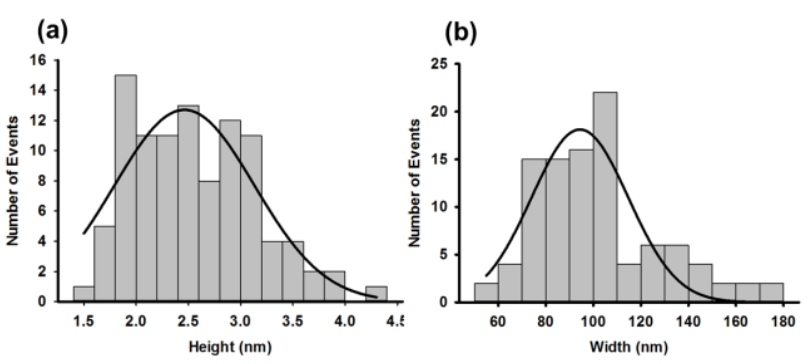

20 Fig. 9. Dimensions of self-assembled moelcular wires. Height distribution (a). Width distribution (b).

\section{Conclusions}

HIV-gp160 reconstituted vesicles could be assembled and visualised to fuse with a supported lipid bilayer at single particle 25 resolution. Although the gp160 protrusions were visible, the morphology was non-spherical unlike the actual HIV virion, suggesting that the protein capsid is necessary for virion stability. Interaction between two parts of the glycoprotein complex, the protruding gp120 and the transmembrane gp41 were shown to be 30 relatively weak, although strong enough to allow self-assembly of molecular wires along a supported bilayer surface. The forces driving the assembly are consistent with the non-covalent interactions thought to dictate the gp120-gp41 complex in vivo. ${ }^{34}$ This work demonstrates that glycoprotein-lipid interactions are 35 capable of driving and influencing assembly and thus should be considered in the development of virosome-based vaccines.

\section{Notes and references}

a Chemical Engineering and Advanced Materials, Newcastle University, 40 Newcastle Upon Tyne.

* Chemical Engineering and Advanced Materials, Newcastle University, Newcastle upon Tyne, NE1 7RU. E-mail: d.j.frankel@newcastle.ac.uk

1 R. C. Aloia, H. Tiant and F. C. Jensen, Proc. Natl. Acad. Sci., 1993, 90, 5181-5185.
M. Moulard, S. Hallenberger, W. Garten and H.-D. Klenk, Virus Res., 1999, 60, 55-65.

C. Fennie and L. A. Lasky, J. Virol. , 1989, 63 , 639-646.

J. M. McCune, L. B. Rabin, M. B. Feinberg, M. Lieberman, J. C. Kosek, G. R. Reyes and I. L. Weissman, Cell, 1988, 53, 5567.

D. C. Chan and P. S. Kim, Cell, 1998, 93, 681-684.

a G. Dalgleish, P. C. Beverley, P. R. Clapham, D. H. Crawford, M. F. Greaves and R. a Weiss, Nature, 1984, 312, 763-767.

J. Huang, B. H. Kang, M. Pancera, J. H. Lee, T. Tong, Y. Feng, H. Imamichi, I. S. Georgiev, G.-Y. Chuang, A. Druz, N. A. Doria-Rose, L. Laub, K. Sliepen, M. J. van Gils, A. T. de la Pena, R. Derking, P.-J. Klasse, S. A. Migueles, R. T. Bailer, M. Alam, P. Pugach, B. F. Haynes, R. T. Wyatt, R. W. Sanders, J. M. Binley, A. B. Ward, J. R. Mascola, P. D. Kwong and M. Connors, Nature, 2014, 515, 138-142.

J.-P. Julien, A. Cupo, D. Sok, R. L. Stanfield, D. Lyumkis, M. C. Deller, P.-J. Klasse, D. R. Burton, R. W. Sanders, J. P. Moore, A. B. Ward and I. a Wilson, Science, 2013, 342, 147783.

A. F. Labrijn, P. Poignard, A. Raja, M. B. Zwick, K. Delgado, M. Franti, J. Binley, V. Vivona, C. Grundner, C.-C. Huang, M. Venturi, C. J. Petropoulos, T. Wrin, D. S. Dimitrov, J. Robinson, P. D. Kwong, R. T. Wyatt, J. Sodroski and D. R. Burton, J. Virol., 2003, 77, 10557-10565.

K. Simons and E. Ikonen, Nature, 1997, 387, 569-572.

D. H. Nguyen and J. E. Hildreth, J. Virol., 2000, 74, 32643272.

M.-P. Mingeot-Leclercq, M. Deleu, R. Brasseur and Y. F. Dufrene, Nat. Protoc., 2008, 3, 1654-1659.

M.-C. Giocondi, V. Vié, E. Lesniewska, P.-E. Milhiet, M. Zinke-Allmang and C. Le Grimellec, Langmuir, 2001, 17, 1653-1659.

4 V. Vié, N. Van Mau, E. Lesniewska, J. P. Goudonnet, F. Heitz and C. Le Grimellec, Langmuir, 1998, 14, 4574-4583.

A. Bitler, N. Lev, Y. Fridmann-Sirkis, L. Blank, S. R. Cohen and Y. Shai, Ultramicroscopy, 2010, 110, 694-700.

R. C. Gallo, P. S. Sarin, E. P. Gelmann, M. Robert-Guroff, E. Richardson, V. S. Kalyanaraman, D. Mann, G. D. Sidhu, R. E. Stahl, S. Zolla-Pazner, J. Leibowitch and M. Popovic, Science, 1983, 220, 865-867.

Y. Kang, K. Guo, B.-J. Li and S. Zhang, Chem. Commun. (Camb)., 2014, 50, 11083-11092.

J. H. Li, Y. F. Wang, W. Ha, Y. Liu, L. S. Ding, B. J. Li and S. Zhang, Biomacromolecules, 2013, 14, 2984-2988.

H. Schönherr, J. M. Johnson, P. Lenz, C. W. Frank and S. G. Boxer, Langmuir, 2004, 20, 11600-11606. 
20 A. Slade, J. Luh, S. Ho and C. M. Yip, J. Struct. Biol., 2002, 137, 283-291.

21 R. Wang, J. Shi, A. N. Parikh, A. P. Shreve, L. Chen and B. I. Swanson, Colloids Surfaces B Biointerfaces, 2004, 33, 45-51.

522 J. L. Hutter and J. Bechhoefer, Rev. Sci. Instrum., 1993, 64, $1868-1873$.

I. Reviakine and A. Brisson, Langmuir, 2000, 16, 1806-1815.

J. Jass, T. Tjärnhage and G. Puu, Biophys. J., 2000, 79, $3153-$ 3163 .

E. J. Bergey, M. I. Cho, M. L. Hammarskjöld, D. Rekosh, M. J. Levine, B. M. Blumberg and L. G. Epstein, Crit. Rev. Oral Biol. Med., 1993, 4, 467-474.

M. Bomsel, D. Tudor, A. S. Drillet, A. Alfsen, Y. Ganor, M. G. Roger, N. Mouz, M. Amacker, A. Chalifour, L. Diomede, G. Devillier, Z. Cong, Q. Wei, H. Gao, C. Qin, G. B. Yang, R. Zurbriggen, L. Lopalco and S. Fleury, Immunity, 2011, 34, 269-280.

28 N. Agrawal, D. P. Leaman, E. Rowcliffe, H. Kinkead, R.

20 Nohria, J. Akagi, K. Bauer, S. X. Du, R. G. Whalen, D. R. Burton and M. B. Zwick, PLoS One, 2011, 6, 1-19.

D. P. Leaman, H. Kinkead and M. B. Zwick, J. Virol., 2010, 84, 3382-3395.

T. Tong, E. T. Crooks, K. Osawa and J. M. Binley, J. Virol., 2012, 86, 3574-3587.

31 V. Buzon, G. Natrajan, D. Schibli, F. Campelo, M. M. Kozlov and W. Weissenhorn, PLoS Pathog., 2010, 6, 1-7.

S. J. Attwood, Y. Choi and Z. Leonenko, Int. J. Mol. Sci., 2013, 14, 3514-3539.

3033 L. Donlon, A. Penaherrera and D. Frankel, Soft Matter., 2013, 9, 2803-2809.

34 R. W. Sanders, M. Vesanen, N. Schuelke, L. Schiffner, R. Kalyanaraman, B. Berkhout, P. J. Maddon, W. C. Olson, M. Lu, J. P. Moore, A. Master and M. Paluch, J. Virol., 2002, 76, 8875-8889. 